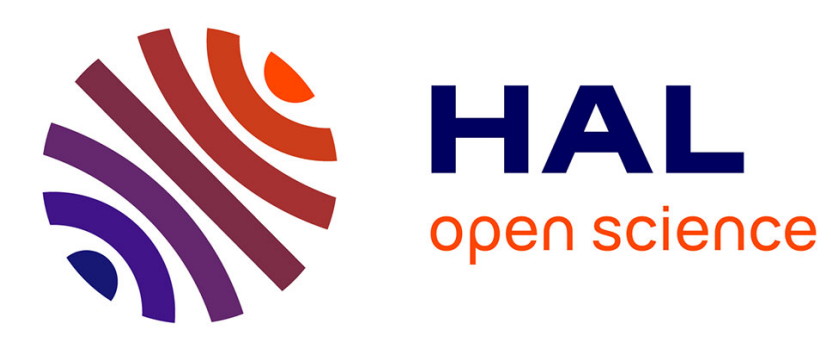

\title{
Pauvreté et mortalité différentielle chez les personnes âgées
}

\author{
Mathieu Lefebvre, Pierre Pestieau, Grégory Ponthière
}

\section{To cite this version:}

Mathieu Lefebvre, Pierre Pestieau, Grégory Ponthière. Pauvreté et mortalité différentielle chez les personnes âgées. 2011. hal-00612606

\section{HAL Id: hal-00612606 \\ https://hal.science/hal-00612606}

Preprint submitted on 22 Aug 2011

HAL is a multi-disciplinary open access archive for the deposit and dissemination of scientific research documents, whether they are published or not. The documents may come from teaching and research institutions in France or abroad, or from public or private research centers.
L'archive ouverte pluridisciplinaire HAL, est destinée au dépôt et à la diffusion de documents scientifiques de niveau recherche, publiés ou non, émanant des établissements d'enseignement et de recherche français ou étrangers, des laboratoires publics ou privés. 


\section{PARISSCHOQL OF ECONOMICS}

WORKING PAPER N² $2011-26$

Pauvreté et mortalité différentielle chez les personnes âgées

Mathieu Lefebvre

Pierre Pestieau

Grégory Ponthière

JEL Codes: I32

Keywords: Powerty measurement, differential mortality, income imputation

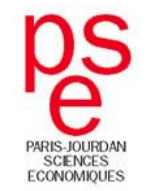




\title{
Pauvreté et mortalité différentielle chez les personnes âgées ${ }^{1}$
}

\author{
Mathieu Lefebvre $^{2}$, Pierre Pestieau ${ }^{3}$ et Gregory Ponthiere ${ }^{4}$
}

\begin{abstract}
Résumé.
Cette note a pour objectif d'illustrer, dans le cas de la Belgique et de ses régions, un problème particulier posé par la mesure de la pauvreté. Etant donné que la mortalité varie selon le niveau de revenu - les personnes aux revenus plus élevés vivant plus longtemps, en moyenne, que les personnes aux revenus plus faibles - les taux de pauvreté calculés pour les classes âgées dépendent non seulement de ce que l'on pourrait appeler la vraie pauvreté, mais aussi de la sélection induite par la mortalité différentielle selon le revenu. En calculant les taux de pauvreté que l'on observerait si des personnes avec différents niveaux de revenus avaient toutes la même espérance de vie, on peut ainsi estimer la vraie pauvreté, en neutralisant les interférences dues à la mortalité différentielle. Cet ajustement des mesures de pauvreté est particulièrement intéressant pour la Belgique, où les écarts de longévité entre Flamands et Francophones et entre riches et moins riches sont importants.
\end{abstract}

Mots-clés: Mesure de pauvreté, mortalité différentielle, revenu imputé.

JEL code: I32

\section{Abstract}

This paper aims at illustrating, in the case of Belgian regions, a specific problem faced by poverty measures. Since mortality is related to the level of income - poor persons tend to die, on average, at younger ages than nonpoor persons - poverty measures for the elderly depend not only on what one can call the "true poverty", but, also, on the selection induced by differential mortality due to income inequality. By computing the hypothetical poverty rates that would prevail if all persons (of all income levels) had the same life expectancy, we estimate new poverty measures, which, by construction, neutralize the interference or the noise due to income-based differential mortality. Such an adjustment of poverty measures is particularly relevant for the case of Belgium, where mortality gaps between Flemish and Walloon, as well as between rich and poor, are sizeable.

Keywords: Poverty measurement, differential mortality, income imputation. JEL code: I32

\footnotetext{
${ }^{1}$ Les auteurs remercient André Decoster et Sergio Perelman pour leurs commentaires.

${ }^{2}$ Université de Liège, CREPP.

${ }^{3}$ Université de Liège, CREPP, CORE, Ecole d'Economie de Paris, et CEPR.

${ }^{4}$ Ecole Normale Supérieure, Paris, et Ecole d'Economie de Paris.
} 


\section{Introduction.}

Dans son Essai sur le Principe de la Population (1798), Thomas Malthus a mis en évidence l'existence de deux types d'ajustements de la taille de la population en présence de pauvreté. D'une part, l'ajustement dit « préventif » de la population, c'est-à-dire la réduction du nombre des naissances par anticipation des difficultés futures posées par l'alimentation et l'éducation des enfants ; d'autre part, l'ajustement dit « positif » de la taille de la population, c'est-à-dire une réduction de la population par la mortalité prématurée des personnes défavorisées. ${ }^{5}$

Etant donné l'état peu développé de la statistique sociale au temps de Malthus, l'affirmation de l'existence des ajustements préventifs et positifs de la population relevait davantage de la conjecture que du résultat scientifique. Cependant, près de deux siècles après l'Essai, plusieurs études empiriques ont confirmé l'existence d'une relation entre niveau de revenu et longévité. Les personnes ayant des revenus élevés vivent, en moyenne, plus longtemps que les personnes ayant des revenus plus faibles. ${ }^{6}$

La mortalité différentielle selon la richesse a des conséquences non négligeables en ce qui concerne la question de la mesure de la pauvreté. Comme cela a été souligné par Kanbur et Mukherjee (2007), les mesures de pauvreté existantes, en se basant sur les populations en vie à un moment donné, reflètent non seulement de ce que l'on pourrait appeler la "vraie » pauvreté, mais, aussi, la sélection induite mécaniquement par la mortalité différentielle. En effet, si la longévité était la même pour tous les niveaux de revenus, il y aurait relativement moins de riches et donc plus de pauvres chez les personnes âgées, en comparaison avec ce qui prévaut en présence d'une mortalité différentielle. La sélection induite par la mortalité différentielle crée donc des interférences (noise) perturbant la mesure de la « vraie » pauvreté.

Dans cet article, nous nous intéressons à l'impact de la mortalité différentielle selon le revenu sur la mesure de la pauvreté, et nous essayons de montrer en quoi le différentiel de mortalité selon la richesse peut expliquer les taux de pauvreté observés au troisième age. Pour ce faire, nous attribuons à toutes les personnes de plus de 60 ans la même espérance de vie; en d'autres termes nous augmentons artificiellement les effectifs observés d'autant plus qu'ils ont de faibles revenus. Cela nous donne un taux de pauvreté corrigé pour les différences de mortalité, que nous comparons ensuite avec le taux de pauvreté non corrigé.

Nous adoptons cette approche pour étudier la pauvreté chez les personnes âgées en Belgique, et dans les deux principales régions belges : la Flandre et la Wallonie. Cet exercice est particulièrement intéressant quand on sait que l'espérance de vie est de près de deux ans plus élevée en Flandre qu'en Wallonie. En d'autres termes, la correction consistera à une double augmentation « artificielle» du nombre de pauvres en Wallonie, pour tenir compte du différentiel entre classes de revenus et du différentiel entre région. Si l'on prend un Wallon appartenant au percentile inférieur, son espérance de vie peut être 5,5 ans plus basse que celle d'un Flamand appartenant au percentile supérieur (2,5 ans pour la région et 3 ans pour les revenus). Or nous savons que le taux de pauvreté observé chez les personnes âgées est plus élevé en Wallonie qu'en Flandre. Cela veut dire que si l'on donnait à tous les mêmes chances de survie, le différentiel de pauvreté Flandre Wallonie serait encore plus élevé.

\footnotetext{
${ }^{5}$ Pour une évaluation des thèses de Malthus à la lumière de l'histoire économique, voir Fogel (1993).

${ }^{6}$ Voir notamment Duleep (1986), Deaton et Paxson (1998), Jusiot (2003) et Salm (2007). Une exception est donnée par Snyder et Evans (2006), qui montrent au contraire que les groupes de revenus plus élevés font face, ceteris paribus, à une mortalité plus grande que celle rencontrée par les groupes moins fortunés.
} 
Pour illustrer le problème qui nous intéresse, prenons une société stationnaire composée de pauvres ayant un revenu annuel de 1 . Les riches ont un revenu annuel de 5. Ils vivent avec certitude jusqu'à 100 ans alors que les pauvres vivent jusqu'à 75 ans. Imaginons qu'au départ les pauvres représentent $80 \%$ des naissances, ce qui veut dire qu'ils représentent $75 \%$ de la population. Si l'on prend l'écart interquintile de l'ensemble de la population, il est égal à 5 . En revanche l'écart interquintile des plus de 75 ans est égal à 1, car pour cette population âgée qui ne comprend plus que des riches (les pauvres étant décédés), le revenu est identique et égal à 5. Cet exemple illustre que le niveau observé d'inégalités des revenus aux âges élevés est, en grande partie, le produit de la mortalité différentielle. En effet, si on faisait vivre artificiellement les pauvres «manquants » jusqu'à 100 ans, on retrouverait l'écart interquintile de 5. Cet article propose précisément d'ajuster les mesures de pauvreté de cette façon, afin de filtrer l'influence des interférences liées à la mortalité différentielle selon le revenu.

Le reste de cette note est organisé comme suit. Dans la Section 2, nous présentons les données sur la pauvreté en 2006 en Belgique et dans ses régions. Nous montrons aussi comment l'espérance de vie varie selon la région, le sexe et le niveau de revenu. Ensuite, dans la Section 3, nous présentons les taux de pauvreté corrigés, et nous les comparons avec les taux de pauvreté habituels. Les conclusions sont tirées en Section 4.

\section{Les données}

Nous utilisons l'enquête Européenne auprès des ménages EU-SILC, ainsi que des estimations de la relation entre niveau d'éducation et espérance de vie réalisées à partir du recensement 2001.

\subsection{Revenu, espérance de vie et pauvreté en Belgique}

Nos estimations vont porter sur l'année 2006. Puisque nous nous intéressons à la pauvreté chez les plus âgés, nous divisons notre échantillon en deux, les moins de 60 ans et les plus de 60 ans. Le Tableau 1 présente le taux de pauvreté par région et par catégorie d'âge.

Le taux de pauvreté est plus élevé dans la population des 60 ans que dans la population totale. Pour l'ensemble du pays, le taux de pauvreté est de $14.2 \%$, tandis que la proportion des personnes pauvres au-delà de 60 ans est de $20.8 \%$. On observe également que, quel que soit la région et le groupe d'âge considéré, le taux de pauvreté est plus élevé chez les femmes que chez les hommes, particulièrement au-delà de 60 ans. Il existe aussi de fortes différences dans les taux de pauvreté entre les différentes régions, en particulier au sein des populations de moins de 60 ans. Après 60 ans, ces différences s'amenuisent, grâce aux systèmes de retraite.

Notons enfin qu'il existe aussi de nettes différences de longévité entre les régions, qui sont pourtant géographiquement très proches. L'espérance de vie à la naissance en Wallonie est plus courte qu'en Flandre d'environ 2 ans et 4 mois. Cet écart n'est pas identique chez les hommes et les femmes : en fait, l'écart d'espérance de vie à la naissance entre Flamands et Wallons est de 1 an et demi chez les femmes, mais de 3 années chez les hommes. 
Tableau 1 : Pauvreté, inégalité et espérance de vie

\begin{tabular}{|c|c|c|c|c|}
\hline & Belgique & $\begin{array}{l}\text { Bruxelles- } \\
\text { capitale }\end{array}$ & Flandre & Wallonie \\
\hline \multicolumn{5}{|l|}{ 1. Taux de pauvreté } \\
\hline Population totale & $14,2 \%$ & $24,4 \%$ & $11,5 \%$ & $16,0 \%$ \\
\hline Hommes & $12,9 \%$ & $23,3 \%$ & $10,1 \%$ & $15,0 \%$ \\
\hline Femmes & $15,4 \%$ & $25,3 \%$ & $12,9 \%$ & $16,9 \%$ \\
\hline $60+$ & $20,8 \%$ & $26,3 \%$ & $20,2 \%$ & $20,4 \%$ \\
\hline Hommes & $18,7 \%$ & $21,6 \%$ & $18,3 \%$ & $18,9 \%$ \\
\hline Femmes & $22,4 \%$ & $29,6 \%$ & $21,9 \%$ & $21,6 \%$ \\
\hline \multicolumn{5}{|c|}{ 2. Distribution du revenu des $60+$} \\
\hline Rapport interquintile & 1,960 & 2,450 & 1,970 & 1,900 \\
\hline Coefficient de Gini & 0,259 & 0,346 & 0,251 & 0,244 \\
\hline Coefficient de variation & 0,566 & 0,758 & 0,536 & 0,545 \\
\hline \multicolumn{5}{|c|}{ 3. Espérance de vie à la naissance } \\
\hline Population totale & 79,4 & 79,1 & 80,2 & 77,9 \\
\hline Hommes & 76,5 & 76,4 & 77,6 & 74,6 \\
\hline Femmes & 82,2 & 81,5 & 82,8 & 81,1 \\
\hline
\end{tabular}

Note : le taux de pauvreté correspond au pourcentage de la population en dessous du seuil de pauvreté évalué à $60 \%$ du revenu médian (seuil $=10236 €$ ).

\subsection{Espérance de vie selon le revenu}

Outre le sexe et l'appartenance géographique, une autre source d'inégalité d'espérance de vie est le revenu. Cependant, l'impact de cette dimension est plus difficile à mesurer, car il n'existe pas de tables de mortalité en fonction du revenu. Dès lors, pour obtenir une relation entre mortalité et revenu, nous sommes passés par des tables de mortalité selon le niveau d'instruction, qui sont régulièrement publiées. ${ }^{7}$ En utilisant la relation revenu-éducation qui peut être estimée, nous avons pu obtenir des tables de mortalité par revenu, région et sexe. ${ }^{8}$

Les Figures 1 et 2 présentent ces estimations pour la classe d'âge 55-59 ans pour les hommes et les femmes séparément. En effet, puisque nous nous intéressons aux 60 ans et plus, nous allons utiliser l'espérance de vie estimée dans la classe d'âge 55-59 ans pour corriger les taux de pauvreté pour l'effet de la mortalité différentielle selon le revenu.

A la lumière des Figures 1 et 2, il apparaît que l'espérance de vie varie significativement en fonction du niveau de revenu, mais d'une manière non linéaire. Par exemple, un Wallon appartenant à la classe de revenu la plus basse a une espérance de vie à 55-59 ans d'environ 4 années de moins qu'un Wallon appartenant à la classe de revenu la plus élevée. Des écarts similaires - quoique plus petits - peuvent aussi être observés pour les femmes (Figure 2).

En somme, les Figures 1 et 2 mettent en évidence l'existence d'écarts d'espérance de vie significatifs selon le niveau de revenu, le sexe et la région. C'est sur base de ces espérances de vie par sexe, niveau de revenu et région que nous allons, dans la section suivante, corriger les

\footnotetext{
${ }^{7}$ Voir Van Oyen et al. (2005) sur la construction de ces tables de mortalité par niveau d'éducation.

${ }^{8}$ Voir l'Annexe pour la construction des Figures 1 et 2.
} 
effectifs de chaque classe de revenu pour les hommes et les femmes séparément, en vue de calculer des mesures de pauvreté ajustées pour la mortalité différentielle selon le revenu.

Figure 1 : Espérance de vie à 55-59 ans par classe de revenu - hommes

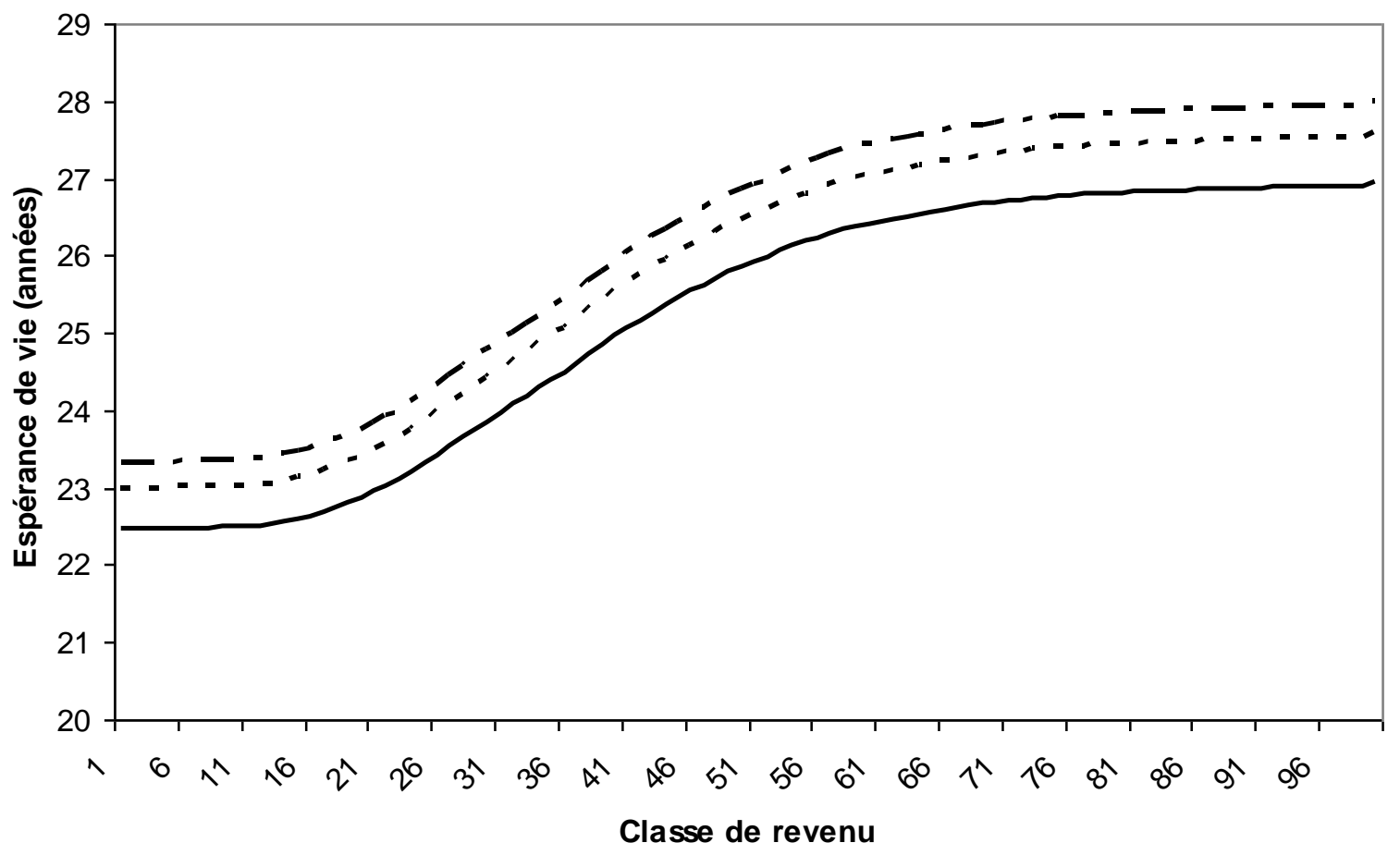

- - - Bruxelles-Capitale - - Flandre — Wallonie 
Figure 2 : Espérance de vie à 55-59 ans par classe de revenu - femmes

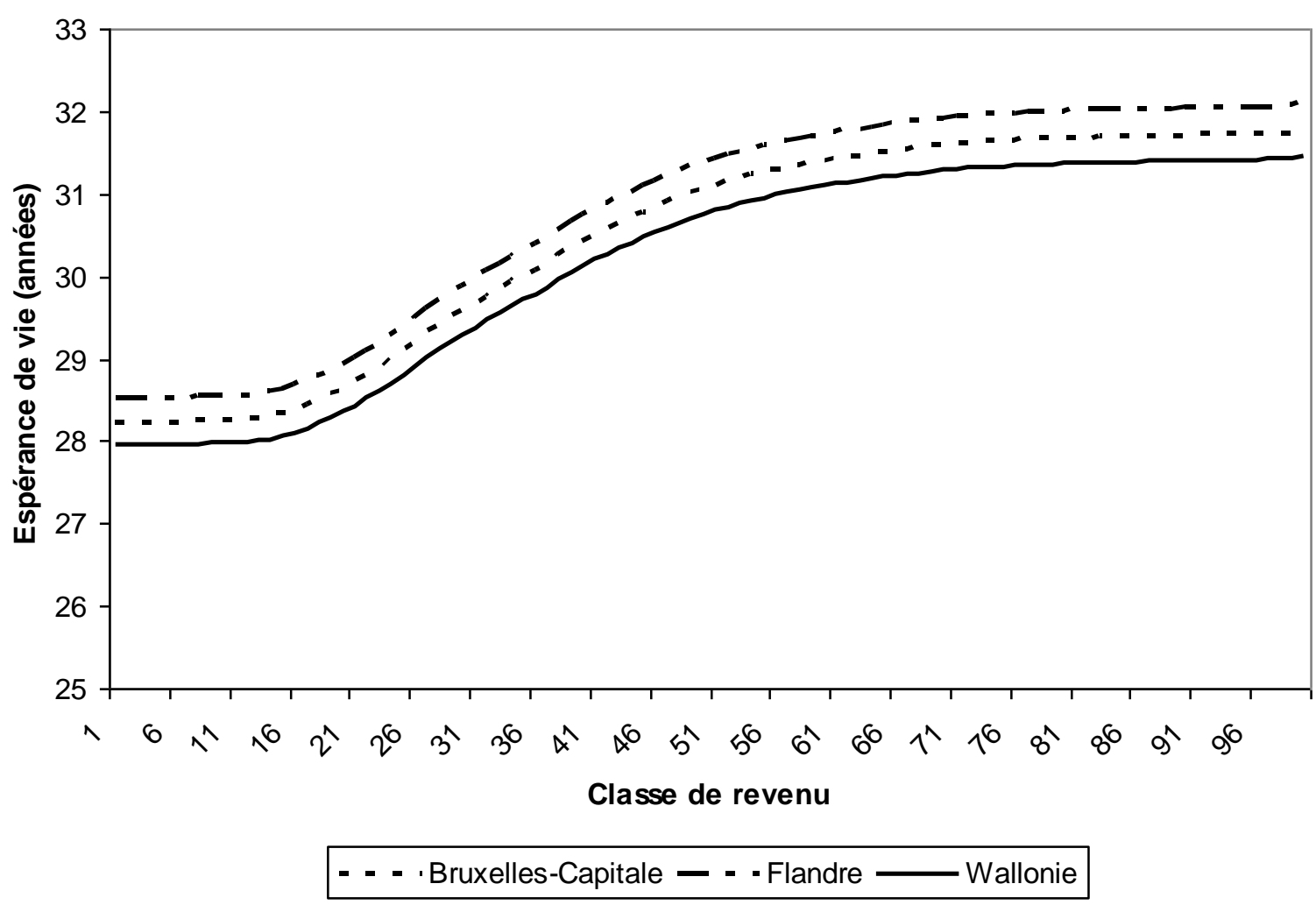

\section{Les taux de pauvreté corrigés}

Afin de corriger les mesures de pauvreté des 60 ans et plus pour les effets de la mortalité différentielle selon le revenu, nous avons procédé comme suit. Pour chacune des classes de revenu $i$ et région $j$, nous avons augmenté l'effectif $N_{i, j}$, en nous basant sur l'espérance de vie $l_{i, j}$ la plus grande observée, c'est à dire celle de la classe de revenus la plus élevée en Flandre (dénotée par $\left.l_{100, f l a}\right)$. Après correction, l'effectif d'une classe $i$ dans la région $j$ est donné par :

$$
N_{i, j}^{\text {new }}=N_{i, j}^{\text {old }} \frac{l_{100, \text { fla }}}{l_{i, j}}
$$

Nous avons ensuite recalculé les taux de pauvreté, et les avons comparés avec les taux de pauvreté originaux. Le Tableau 2 présente les taux de pauvreté après correction, pour la Belgique, ainsi que pour la Flandre et la Wallonie. Nos résultats seront présentés en deux temps. Dans un premier temps, nous allons montrer des taux de pauvreté corrigés sous l'hypothèse que l'on garde le même seuil de pauvreté qu'avant la correction. Dans un deuxième temps, nous montrerons les taux de pauvreté ajustés lorsque l'on utilise le nouveau seuil de pauvreté associé à la nouvelle distribution de revenu.

Lorsqu'on garde l'ancien seuil de pauvreté (10236 €), le taux de pauvreté chez les 60 ans et plus augmente dans toutes les régions suite à notre correction. Ceci n'est pas surprenant, car notre correction a consisté en quelque sorte à ajouter des personnes pauvres «manquantes » du fait de la mortalité différentielle selon le revenu. Cette augmentation relative des effectifs plus pauvres doit forcément impliquer une hausse des taux de pauvreté. Il est par contre étonnant d'observer que la hausse du taux de pauvreté est approximativement la même pour tous les groupes considérés, et correspond à environ 1 point. Il existe cependant des variations entre les divers groupes. Les hommes wallons de plus de 60 ans sont ceux chez qui 
l'ajustement des taux de pauvreté est le plus grand : alors qu'ils connaissaient une pauvreté de $18.9 \%$ avant correction, après correction, ce taux passe à $20.1 \%$. La correction est relativement plus petite au sein des autres groupes.

La première partie du Tableau 2 semble ainsi suggérer que les mesures habituelles de la pauvreté tendent à sous-estimer l'ampleur de la pauvreté au troisième âge, dans une mesure qui dépend de l'importance de la mortalité différentielle selon le revenu. La correction effectuée entraîne une hausse des taux de pauvreté, qui correspond en moyenne à environ un point dans tous les cas de figure. Cet ajustement d'environ $1 \%$ est à la fois peu et beaucoup. Peu par rapport à des taux de pauvreté tournant autour de $20 \%$. Beaucoup quand on pense au nombre de familles qui seraient tombées dans la pauvreté si leurs membres avaient vécu aussi longtemps que les personnes plus riches.

Alors que la première partie du Tableau 2 repose sur un seuil de pauvreté identique à celui qui prévalait avant la correction, il est important de signaler que la modification des effectifs en vue de neutraliser l'effet de la mortalité différentielle sur la mesure de la pauvreté a eu aussi comme effet de modifier la distribution du revenu dans la population. Dès lors, si l'on adhère à une conception relativiste - plutôt qu'absolutiste - de la pauvreté, le seuil de pauvreté doit être recalculé sur base de cette nouvelle distribution du revenu. Si l'on recalcule le seuil de pauvreté sur base des nouveaux effectifs, on observe que le seuil de pauvreté baisse de $125 €$.

Tableau 2 : Pauvreté corrigée chez les 60+

\begin{tabular}{lccc}
\hline & Belgique & Flandre & Wallonie \\
\hline & \multicolumn{1}{c}{ l. Taux de pauvreté } & \\
& Ancien seuil de pauvreté $(10236 €)$ & \\
Population totale & $21,7 \%(+0.9)$ & $21,1 \%(+0.9)$ & $21,3 \%(+0.9)$ \\
Hommes & $19,8 \%(+0.9)$ & $19,3 \%(+1.0)$ & $20,1 \%(+1.2)$ \\
Femmes & $23,3 \%(+0.9)$ & $22,7 \%(+0.8)$ & $22,3 \%(+0.7)$ \\
& & & \\
& & & \\
Population totale & Nouveau seuil de pauvreté $(10109 €)$ & $20,0 \%(-0.4)$ \\
Hommes & $20,5 \%(-0.3)$ & $20,2 \%(\mathrm{cst})$ & $18,4 \%(-0.5)$ \\
Femmes & $18,7 \%(\mathrm{cst})$ & $18,3 \%(\mathrm{cst})$ & \\
& $22,1 \%(-0.3)$ & $21,8 \%(-0.1)$ & $21,2 \%(-0.4)$ \\
Rapport interquintile & & & \\
Coefficient de Gini & $1,940(-0,020)$ & $1,960(-0,010)$ & $1,860(-0,040)$ \\
Coefficient de variation & $0,255(-0,004)$ & $0,248(-0,003)$ & $0,240(-0,004)$ \\
& $0,559(-0,007)$ & $0,531(-0,005)$ & $0,536(-0,006)$
\end{tabular}

Note : le taux de pauvreté correspond au pourcentage de la population en dessous du seuil de pauvreté évalué à $60 \%$ du revenu médian. La variation par rapport aux chiffres avant correction est présentée entre parenthèse.

Cette modification du seuil de pauvreté, qui passe à $10109 €$, est loin d'être neutre. Comme cela est montré dans la deuxième partie du Tableau 2, cette baisse du seuil de pauvreté, induite par la nouvelle distribution du revenu suite à nos corrections, a pour effet de modifier le taux de pauvreté à la baisse, quoique très légèrement et quasiment pas du tout en Flandre. Enfin, si on se tourne vers les indicateurs d'inégalité de la distribution du revenu, on remarque que les trois indicateurs tendent vers une distribution moins inégale, avec une diminution plus forte en Wallonie qu'en Flandre. 


\section{Conclusions}

La statistique sociale et économique est sujette à des interférences générées par la mortalité différentielle. En effet, on ne peut compter, quantifier et calculer que pour les personnes qui sont présentes dans la population, et pas pour les «absents ». Dans la mesure où les diverses sous-populations étudiées ne sont pas sujettes aux mêmes conditions de survie, cette emphase sur les «présents » peut être source de sérieux problèmes d'estimation et d'interprétation. La mesure de la pauvreté n'échappe pas à ce problème : vu que les personnes aux revenus faibles connaissent, en moyenne, des conditions de survie moins favorables que les personnes aux revenus élevés, les taux de pauvreté sont influencés par cette mortalité différentielle.

Le but de cet article était d'illustrer, pour le cas de la Belgique et de ses régions, ce problème rencontré par la mesure de la pauvreté. Pour ce faire, nous avons recalculé les taux de pauvreté pour les 60 ans et plus, sous l'hypothèse d'une mortalité identique pour tous les niveaux de revenu. Les taux de pauvreté ainsi calculés excèdent les taux habituels par, en moyenne, un point de pourcentage, ce qui est loin d'être négligeable. Cependant, si le seuil de pauvreté est lui aussi corrigé en fonction de la prise en compte des personnes pauvres «manquantes », alors les taux de pauvreté reviennent à des niveaux proches de ceux observés avant la correction. Dès lors la mesure dans laquelle les taux de pauvreté habituels sousestiment la pauvreté « vraie » dépend de notre conception de la pauvreté : absolue ou relative.

En somme, cet article montre que l'alignement de la longévité des pauvres sur celle des riches n'entraine pas de fortes différences dans la mesure de la pauvreté. D'aucuns pourraient s'interroger sur l'intérêt et la pertinence de cet exercice. Plusieurs remarques peuvent être faites à ce sujet. Tout d'abord, les résultats auraient été différents si les pauvres avaient disposé de moins de ressources au cours de leur «supplément de vie » (dans notre cas, le revenu imputé était celui qu'elles avaient de leur vivant). ${ }^{9}$ Ensuite, il est vraisemblable que, dans les pays en développement où les différences de longévité sont plus fortes, les résultats auraient été plus marquants. Finalement, ce n'est pas parce que les écarts mesurés sont minimes que le problème n'est pas intéressant. Si le taux de pauvreté avait doublé du fait de cet allongement artificiel de la durée de vie, on aurait alors trouvé le problème intéressant, car démontrant un manque de robustesse des mesures de pauvreté. Nos calculs illustrent au contraire une certaine robustesse de ces mesures, robustesse qui n'était pas évidente a priori.

\section{Annexe : Tables de mortalité par classe de revenu}

Nous ne disposons pas de tables de mortalité par niveau de revenu en Belgique, mais par contre nous disposons des tables de mortalité par niveau d'instruction (Deboosere et al., 2009). A partir de ces informations, il est possible d'estimer des tables de mortalité par classes de revenu au moyen d'une méthode de régression pondérée, comme dans l'étude réalisée par Bossuyt et Van Oyen (2000). Pour ce faire, on effectue une régression linéaire sur les prévalences spécifiques pour l'âge et l'éducation (Pamuk, 1985). Après avoir pris comme hypothèse le fait que les niveaux croissants d'instructions reflètent une hiérarchisation similaire à celle du revenu, on estime un modèle par moindres carrés ordinaires dans lequel la variable dépendante est le taux de mortalité pour chaque niveau d'instruction cumulé de manière croissante. Une fois estimés, les paramètres de cette fonction peuvent être utilisés pour reconstruire des tables de mortalité par classe de revenu.

\footnotetext{
${ }^{9}$ L'ajustement des mesures de pauvreté pour neutraliser les interférences liées à la mortalité différentielle est en effet sensible au niveau du revenu fictif imputé aux personnes « manquantes » (voir Lefebvre et al. 2011).
} 
Notons que dans notre cas nous utilisons des tables de mortalité par classe d'âge de cinq ans afin de garantir une représentation suffisante de chacune de nos classes de revenu. En effet nous divisons notre population en classe de revenu de $500 €$ avec une classe maximale qui englobe tous les revenus au-delà de $50000 €$.

\section{Références}

Bossuyt, N. et H. Van Oyen (2000), Espérance de vie en bonne santé selon le statut socioéconomique en Belgique, Institut de la Santé Publique, Bruxelles.

Deaton, A. \& Paxson, C. (1998): "Aging and inequality in income and health", American Economic Review, 88, pp. 248-253.

Deboosere, P, Gadeyne, S. \& Van Oyen H (2009): “The 1991-2004 Evolution in Life Expectancy by Educational Level in Belgium Based on Linked Census and Population Register Data", European Journal of Population, 25, pp. 175-196.

Duleep, H.O. (1986): "Measuring the effect of income on adult mortality using longitudinal administrative record data", Journal of Human Resources, 21 (2), pp. 238-251.

EU-SILC (2006): “Statistics on Income and Living Conditions”, European Commission.

Fogel, R. (1993): "Economic growth, population theory and physiology: the bearing of longterm processes on the making of economic policy", American Economic Review, 84 (3), pp. 369-395.

Jusiot, F.(2003) : "Inégalités sociale de mortalité : effet de la pauvreté ou de la richesse", miméo.

Kanbur, R. \& Mukherjee, D. (2007): "Premature mortality and poverty measurement", Bulletin of Economic Research, 59 (4), pp. 339-359.

Lefebvre, M., Pestieau, P. \& Ponthiere, G. (2011): "Measuring poverty without the mortality paradox", mimeo.

Malthus, T. (1798): An Essay on the Principle of Population, London.

Pamuk E. (1985): "Social class inequality in mortality from 1921 to 1972 in England and Wales, Population Studies, 39, 17-31.

Salm, M. (2007): "The effect of pensions on longevity: evidence from Union Army veterans", IZA Discussion Paper 2668.

Snyder, S. \& W. Evans (2006): "The effect of income on mortality: evidence from the social security notch", Review of Economics and Statistics, 88 (3), pp. 482-495.

Van Oyen, H., Bossuyt, N., Deboosere, P., Gadeyne, S., Abatith, E. \& Demarest, S. (2005): "Differential inequity in health expectancy by region in Belgium", Soz.-Präventivmed, 50, pp. 301-310. 